\title{
Experimental investigation on unstable flow in multi-stage centrifugal blower with inlet guide vane
}

\author{
Kiyotaka HIRADATE*, Kiyohide SAKAMOTO*, \\ Yasushi SHINKAWA**, Satoshi JOUKOU** and Takeshi UCHIYAMA** \\ ${ }^{*}$ Center for Technology Innovation - Mechanical Engineering, Research \& Development Group, Hitachi, Ltd. \\ 832-2 Horiguchi, Hitachinaka, Ibaraki 312-0034, Japan \\ E-mail: kiyotaka.hiradate.kf@hitachi.com \\ ${ }^{* *}$ Machinery System Division, Industrial Products Company, Hitachi, Ltd. \\ 603 Kandatsu, Tsuchiura, Ibaraki 300-0013, Japan
}

Received 14 October 2015

\begin{abstract}
This study experimentally investigates the unstable flow in a five-stage centrifugal blower with inlet guide vanes (IGVs) upstream of the first stage. Performance characteristics of the blower were obtained under three IGVs opening conditions. Measurements of the unsteady pressure were performed using six high-response pressure transducers. To distinguish rotating stall from surge, three of these transducers were located thirty degrees apart in the circumferential direction at the vaneless space upstream of the diffuser vane. Three types of pressure fluctuation in the low flowrate region were confirmed. The first one occurred in the positive slope region of the total-system head curve with the largest amplitude and the lowest frequency under the full opening condition of IGVs. The second one was the spike-like fluctuation propagating in the circumferential direction in the vaneless space. The last one appeared under the partial opening condition of IGVs with smaller amplitude and higher frequency than those of the first one. It was confirmed that this pressure fluctuation oscillated at the same phase in the vaneless space and occurred even in the steeply negative slope region of the total-system head curve under the minimum opening condition of IGVs. It was considered that the first one was deep surge and the second one was rotating stall. The last one was considered to be mild surge. Under the minimum opening condition of IGVs, the slope of the head curve of the first stage becomes steeply negative by prewhirl. This negative slope stabilizes the total system of the blower. By contrast, the slope of the head curve of the other stages becomes positive in the low flowrate region. This positive slope destabilizes the total system of the blower. Therefore, even if the total-system head curve keeps a steeply negative slope, the system operating point is considered to oscillate slightly, corresponding to mild surge situation, due to these stability balances.
\end{abstract}

Key words : Centrifugal blower, Multi-stage, Inlet guide vane, Rotating stall, Surge

\section{Introduction}

Centrifugal blowers and compressors are widely used for the gas compression in various industrial fields. Higher efficiency and wider operating range are required for these machines. The operating range of these machines is strongly affected by unstable flow phenomena, i.e., rotating stall and surge. Therefore, investigating the inception mechanism and flowfield of rotating stall and surge is very important to improve the operating range of these machines.

Much research about rotating stall and surge in single-stage centrifugal turbomachinery has been conducted. As for rotating stall, a lot of studies related to both the vaneless and the vaned diffuser have been reported. For example, Senoo et al. experimentally and analytically investigated a relation between the reverse flow and the inception of rotating stall in a vaneless diffuser of a centrifugal blower. They proposed criteria for the inception of rotating stall in vaneless diffusers (Senoo, et al., 1977; Senoo, et al., 1978). Spakovszky et al. investigated the inception of rotating stall in a centrifugal compressor with a vaned diffuser (Spakovszky and Roduner, 2007). They confirmed two patterns of the 
inception that were well known in axial compressors. One was the short wavelength inception, so called "spike", in the vaneless space between the impeller and vaned diffuser. The other was the long wavelength inception, so called "modal wave", in the same vaneless space. Moreover, Spakovszky et al. suggested that the spike or the modal wave was the sign of the instability, which appeared depending on the relation between the slope of pressure-rise characteristic in the semi-vaneless space and that in the overall diffuser. Joukou et al. confirmed the spike even in a low solidity vaned diffuser (LSD) of a centrifugal blower (Joukou, et al., 2012). They indicated that the reverse flow existed near hub wall in the vaneless space between the impeller and vaned diffuser when the spike occurred. Moreover, they confirmed that LSD with smaller radius of leading edge could suppress this reverse flow and an occurrence of spike.

With regard to surge, a lot of studies have been executed in combination of detailed unsteady pressure and velocity measurements and a theoretical prediction model of the system dynamics, i.e., a nonlinear lumped-parameter model, which was proposed by Greitzer for axial compressors (Greitzer, 1976a, 1976b). Hansen et al. compared experimental results of surge in a single-stage centrifugal compressor with predictions using the Greitzer model and confirmed the applicability of the model for the centrifugal compressor (Hansen, et al., 1981). Fink et al. measured the flowfield of a single-stage centrifugal compressor from the surge inception to during surge. In addition, they succeeded to improve the prediction accuracy of the system dynamics during surge by including the variation of impeller rotation speed in the Greitzer model (Fink, et al., 1992).

Multi-stage centrifugal turbomachineries are often applied in case that high discharge gas pressure with relatively low rotor speed is required. There are several literatures about unstable flow phenomena in this type of machines. For example, Arnulfi et al. measured the unsteady flowfield from before to during rotating stall in a two-stage and a four-stage centrifugal bower with vaneless diffuser (Arnulfi, et al., 1996). Arnulfi et al. also performed measurements of unsteady pressure and velocity during rotating stall and surge in a four-stage centrifugal blower with vaned diffusers. They compared measurement results with theoretical predictions of the system dynamics using the Greitzer model and confirmed that the Greitzer model was accurate enough to describe quantitatively the system dynamics of the multi-stage centrifugal blower (Arnulfi, et al., 1999a, 1999b).

In multi-stage centrifugal turbomachineries, inlet guide vanes (IGVs) for generating prewhirl are often equipped upstream of a first-stage impeller to reduce the shaft power and improve the operating range under the part-load condition. However, there are only a few studies examining effects of IGVs on the performance of these types of machines, e.g., a report conducted by Lüdtke (Lüdtke, 1992). Moreover, as far as we know, there are no literatures investigating unstable flow phenomena in these types of machines in detail.

In this paper, we measured the unsteady pressure fluctuation in a five-stage centrifugal blower, in which IGVs were installed upstream of the first stage, to clarify what kinds of unstable flow phenomena occurred. We installed high response pressure transducers on the blower casing and measured the pressure fluctuation at several flowrates changing the IGVs from the full opening to partial opening state. We confirmed three kinds of pressure fluctuations in low flowrate region and considered what kinds of unstable flow phenomena caused these pressure fluctuations.

\section{Nomenclature}

$C_{p}=\Delta P s s / 0.5 \rho_{0} U_{2}^{2}$
$C_{p}{ }^{2}=\Delta P s u / 0.5 \rho_{0} U_{2}^{2}$
$D$
$F$
$H$
$P s s$
$P s u$
$Q$
$T$
$U$
$f$
$g$
$t$
$\Psi=H_{a d} / U_{2}^{2} / g$
$\beta$
$\phi=4 Q / \pi D_{2}^{2} U_{2}$
$\rho$

static pressure-rise coefficient [-]

static pressure fluctuation coefficient [-]

diameter [m]

impeller rotation frequency $[\mathrm{Hz}]$

head $[\mathrm{m}]$

steady static pressure $[\mathrm{Pa}]$

unsteady static pressure [Pa]

inlet volume flow $\left[\mathrm{m}^{3} / \mathrm{s}\right]$

period of impeller rotation [s]

impeller peripheral speed $[\mathrm{m} / \mathrm{s}]$

frequency $[\mathrm{Hz}]$

gravitational acceleration $\left[\mathrm{m} / \mathrm{s}^{2}\right]$

time [s]

adiabatic head coefficient [-]

blade angle from tangential direction [degree]

flow coefficient [-]

density $\left[\mathrm{kg} / \mathrm{m}^{3}\right]$ 


$\begin{array}{cl}\text { Subscripts } & \\ \text { ad } & \text { adiabatic } \\ \text { all } & \text { total system } \\ d & \text { diffuser } \\ \text { des } & \text { design flowrate } \\ i & \text { impeller } \\ s & \text { suction } \\ 0 & \text { atmospheric condition } \\ 2 & \text { impeller outlet }\end{array}$

$\begin{array}{cl}\begin{array}{c}\text { Abbreviations } \\ \text { IGVs }\end{array} & \text { inlet guide vanes } \\ \text { IOR } & \text { IGVs opening ratio } \\ \text { LSD } & \text { low solidity vaned diffuser } \\ \text { RTC } & \text { return channel }\end{array}$

\section{Experimental procedure}

\subsection{Experimental apparatus}

Figure 1 shows a schematic diagram of meridional section of a multi-stage centrifugal blower investigated in this study. This blower is used for sewage aeration and consists of a suction nozzle, an IGVs, five centrifugal blower stages composed of a centrifugal impeller, a LSD and a return channel (RTC) in each stage, and a discharge scroll. These blower stages are designed to satisfy the similarity condition of the inner flow. For example, the incidence, deceleration ratio and turning angle of each stage are set to the same value at the design flowrate. Main specifications of the blower are listed in Table 1.

The IGVs has 12 straight blades and the blade setting angle of the IGVs can be changed by a link mechanism. The impellers are full-shrouded type and its outlet diameter $D_{2}$ are $820 \mathrm{~mm}$. Each impeller has 15 three-dimensional back-swept blades whose blade outlet angles $\beta_{2}$ are 40 degree from tangential direction. The LSDs consist of parallel straight walls and 12 vanes with NACA63 airfoil profile. The RTCs have 14 blades with NACA63 airfoil profile.

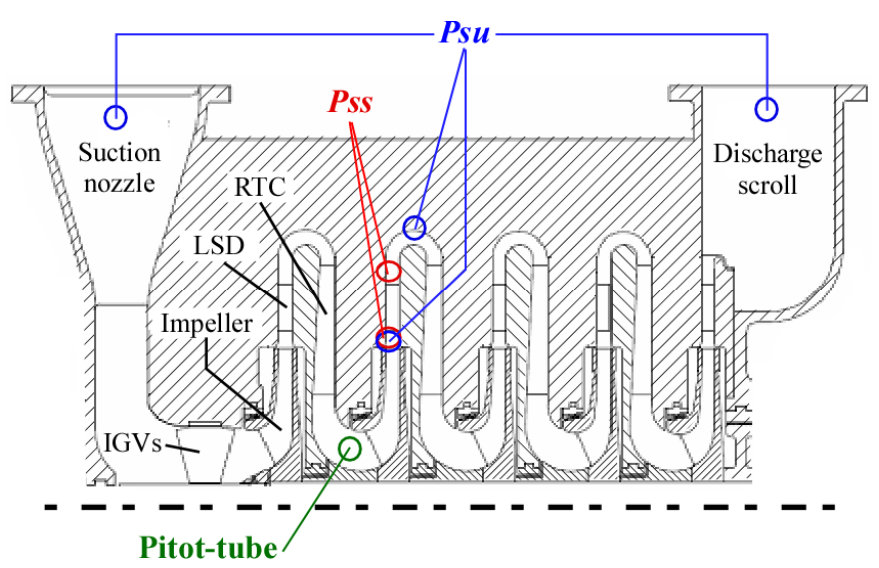

Fig. 1 Schematic diagram of tested blower.
Table 1 Main specifications of tested blower.

\begin{tabular}{|c|c|c|}
\hline $\begin{array}{c}\text { Suction flow coefficient } \\
\text { at design flowrate }\end{array}$ & $\phi_{s_{-} \text {des }}$ & 0.040 \\
\hline Shaft rotational speed & $N\left(\mathrm{~min}^{-1}\right)$ & 2969 \\
\hline Number of stages & - & 5 \\
\hline
\end{tabular}




\subsection{Experimental method}

In this study, experiments were carried out at three IGVs opening ratios (IOR), 100\% (full opening), 10\% (partial opening) and 4.5\% (minimum opening). Under each IOR condition, the volumetric flowrate of the blower was gradually decreased by throttling a valve located in a discharge piping downstream of the discharge scroll, and measurements of the steady-state performance and the unsteady flowfield were conducted.

The measurements of the steady-stage performance of the blower were conducted as follows. The volumetric flowrate of the blower was measured by an orifice plate placed in a suction piping upstream of the suction nozzle. The total system adiabatic head was derived from total pressure and total temperature measured at the suction and the discharge piping. Seven five-hole pitot-tubes were located at the inlet of the second stage impeller as shown green circles in Fig. 1 and Fig. 2. In Fig. 2, all these pitot-tubes are drawn to be set between two adjacent RTC blades. However, in fact, each probe was installed downstream of a different blade-to-blade passage of the RTC to minimize an effect of these probes on the flowfield. The effect of these probes on the flowfield will be discussed in the later section. Moreover, 14 steady static pressure ( $P s s)$ taps were equipped in the second-stage diffuser as shown in Fig. 3. Here, "steady" means "the state where the time variation was eliminated by time-averaging." These pitot-tubes and Pss taps enable us to obtain the static pressure-rise in the first stage (from the suction nozzle inlet to the first stage outlet) and that in the second stage (from the inlet of the second stage to the outlet of the second stage diffuser) separately. The static pressure-rise in the second stage represents those in the subsequent stages since all blower stages satisfy the similarity condition of the inner flow as mentioned above. Calibration curves ranging from -40 degree to +40 degree of both yaw-angle and pitch-angle for these pitot-tubes were obtained in advance. In the measurements, tips of the pitot-tube probes were fixed parallel to the impeller rotation axis. The static pressure, dynamic pressure, and absolute flow angle at the inlet of the second stage were calculated by the calibration curves.

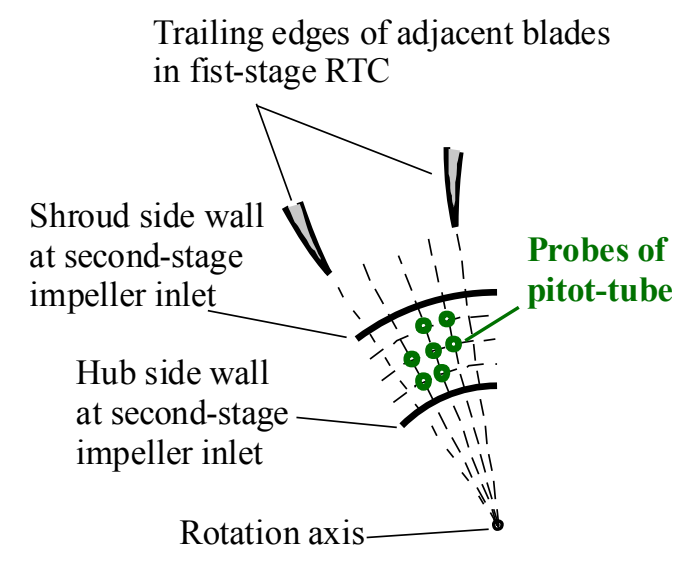

Fig. 2 Location of probes of pitot-tube

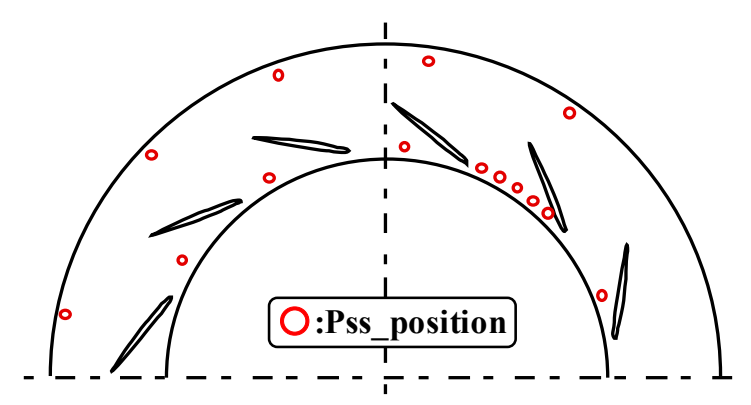

Fig. 3 Location of static pressure taps in LSD

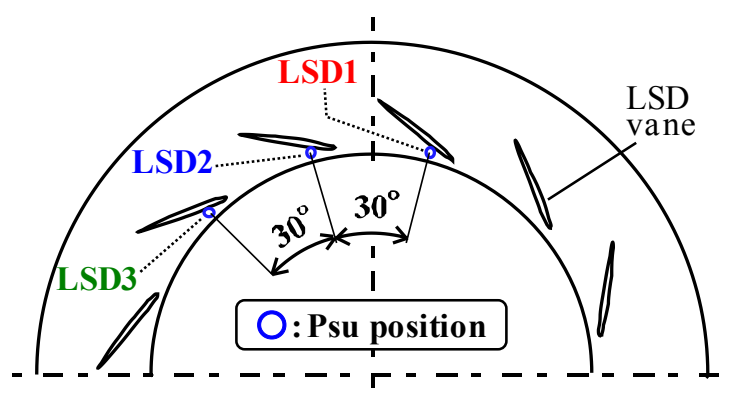

Fig. 4 Location of high-response pressure transducers 
As for the unsteady flowfield, the measurements of the unsteady static pressure (Psu) were performed. Six high-response pressure transducers (Kulite XT-190A-5A) were installed in the blower casing. In Fig. 1, installation positions of these pressure transducers are also indicated by blue circles. Since this blower was actual commercial product to be delivered to a customer, the positions where the transducers could be installed were very limited. The installation positions of these pressure transducers were selected so that no additional machining of the blower casing was needed and the instrumentation could be achieved only using several holes and hollow spaces that originally existed in the blower casing. As shown in Fig. 1, these pressure transducers were mounted on the suction nozzle, an U-turn bend between the LSD and the RTC in the second stage, and the discharge nozzle to detect surge. In addition, as shown in Fig. 4, three pressure transducers (LSD1, LSD2, and LSD3) were mounted 30 degrees apart on the shroud wall at the vaneless space near the leading edge of the LSD in the second stage to distinguish rotating stall in the diffuser from surge. As mentioned above, this blower was design so that the all blower stages satisfy the similarity condition of the inner flow. Therefore, it was considered that the rotating stall in the vane less space should have appeared similarly in all stages if it occurred. In addition, surge has the one-dimensional propagation characteristic of the pressure fluctuation and could be measured anywhere in the blower. Thus, this instrumentation of the pressure transducers was thought to be enough to achieve the aim of this experiment. Sampling frequency of the pressure transducers was $1.5 \mathrm{kHz}$ and recorded $P s u$ data were analyzed by using a FFT analyzer.

\section{Results and discussion}

Figure 5 shows measured total system head-flow characteristics at each IOR. The horizontal axis means the suction flow coefficient $\phi_{s}$ derived from the volumetric flowrate at the orifice plate and the vertical axis means the total system adiabatic head coefficient $\Psi_{\text {ad_all }}$. Measurement points at the smallest flowrate at IOR $100 \%$ and $10 \%$ corresponded to the safety operation limit just before dangerous, large amplitude pressure oscillations took place. On the other hand, a measurement point at the smallest flowrate at IOR 4.5\% was not the safety operation limit but a point where no more clearly changes of the $P s u$ fluctuation could be observed with closing the throttle valve.

In Fig. 5, total system head-flow characteristics at IOR $100 \%$ and $10 \%$ in case without the pitot-tubes in Fig. 2 are also indicated by red circles. The total system head-flow characteristics in case without the pitot-tubes agreed well with those with the pitot-tubes. In particular, the suction flow coefficients at the safety operation limit were perfectly consistent between these two cases. Therefore, we considered that the effect of the pitot-tubes on the flowfiled in the low flowrate region was negligible.

In the following section, we will show measured $P s u$ time history and FFT analysis results under each IOR condition.

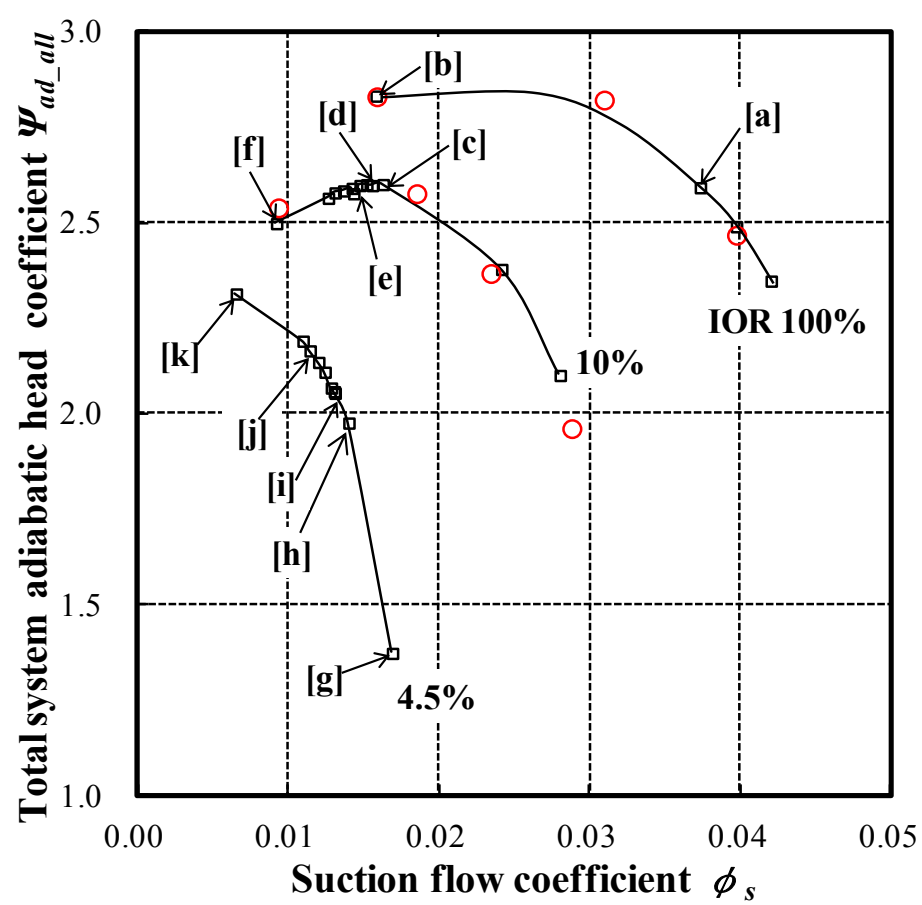

Fig. 5 Total system head-flow characteristics. 


\subsection{Measurement results at IOR $100 \%$}

Figures 6 and 7 show measured Psu fluctuations of each positions at IOR 100\%. Figure 6 shows Psu time histories at points [a] and [b] in Fig. 5 and lower flowrate than point [b]. The horizontal axis means the time normalized by a period of the impeller rotation $(t / T)$. The vertical axis means the static pressure fluctuation coefficient $C p$ ' normalized by dynamic pressure derived from the atmospheric density $\rho_{0}$ and the peripheral speed of the impeller outlet $U_{2}$. Figure 7 shows time-averaged $P s u$ fluctuation spectra at the inlet of the suction nozzle, the LSD1, and the outlet of the discharge scroll. The horizontal axis means the frequency normalized by the impeller rotation frequency $(f / F)$. The vertical axis means $P s u$ fluctuation level divided by a base pressure $(1 \mathrm{kPa})$.

As shown in Figs. 6 and 7, no remarkable Psu fluctuations were observed at the operating point [a] in Fig. 5. However, when the operating point reached to the safety operation limit [b] where the slope of $\Psi_{a d}$ all was supposed to be almost flat, weak Psu fluctuations and peak frequencies began to appear at all the Psu measurement positions. The peak frequencies of these fluctuations were $8.9 \%$ of the impeller rotation frequency. When the valve was closed slightly more, the amplitudes of the $P s u$ fluctuations became larger suddenly. The peak frequencies of these fluctuations became smaller than those observed at the operating point [b], i.e., $7 \%$ of the impeller rotation frequency.
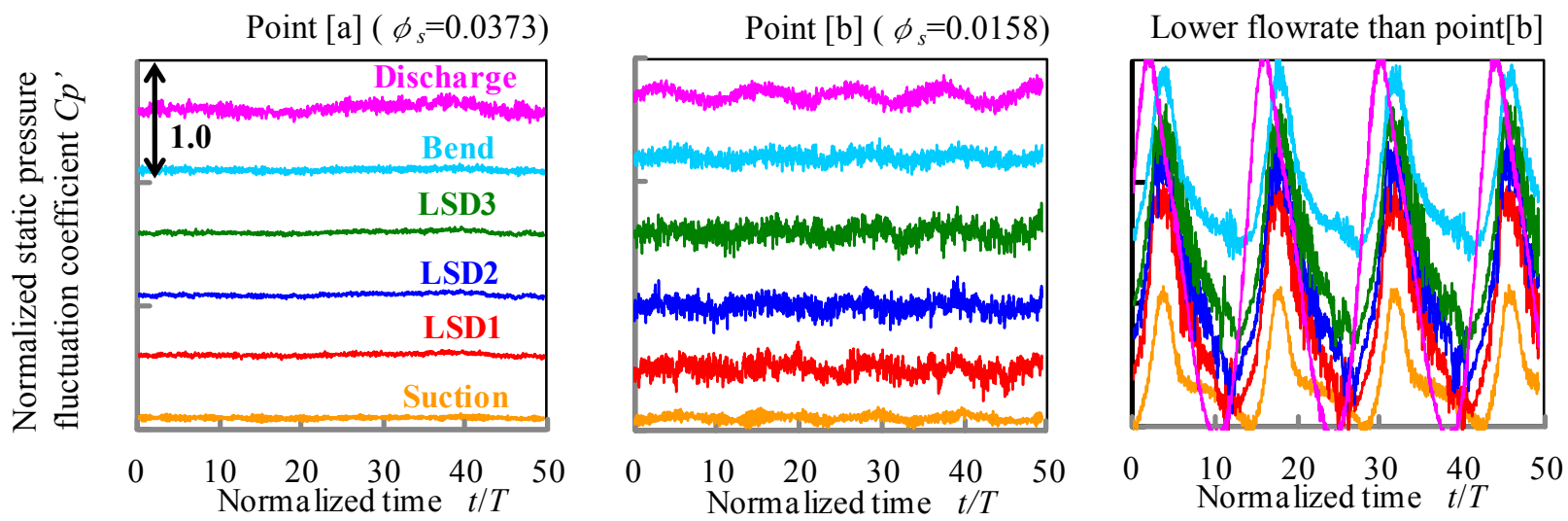

Fig. 6 Time history of $P s u$ fluctuations at IOR $100 \%$

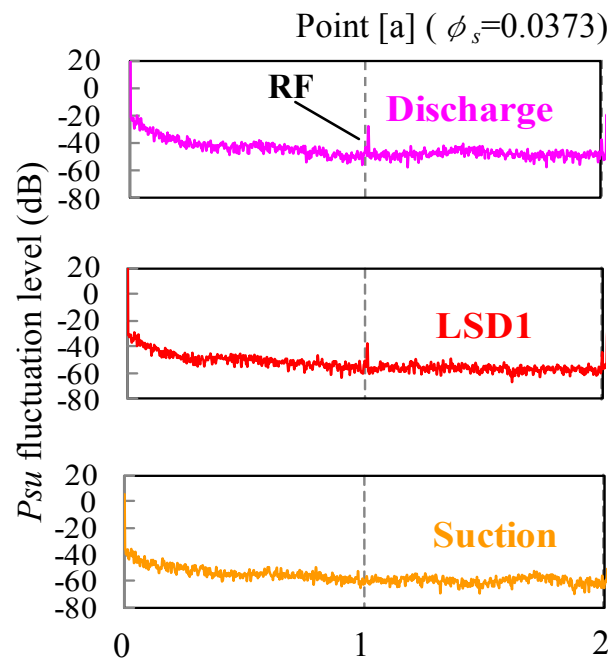

Normalized frequency $f / F$
Point $[\mathrm{b}]\left(\phi_{s}=0.0158\right)$
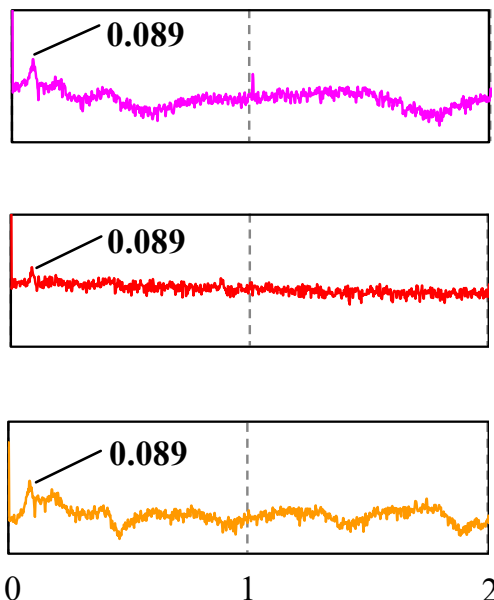

Normalized frequency $f / F$
Lower flowrate than point[b]
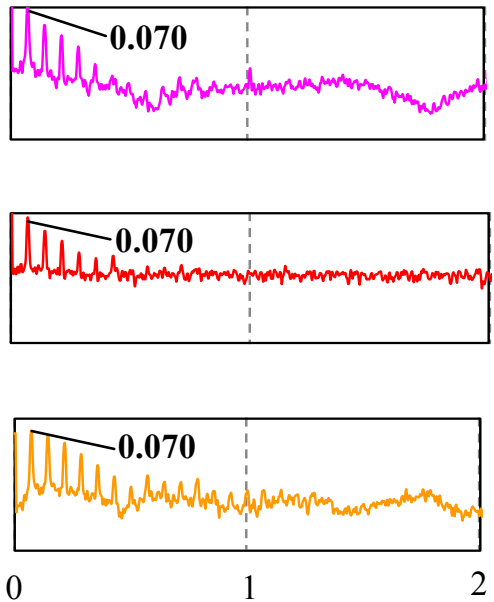

Normalized frequency $f / F$

Fig. 7 Time-averaged Psu fluctuation spectra at IOR 100\% 


\subsection{Measurement results at IOR $10 \%$}

Figures 8 and 9 show measured $P$ su fluctuations at IOR 10\%.

At the operating point [c] in Fig. 5, no remarkable Psu fluctuations were observed. When the valve was throttled and the operating point reached the point [d] where the slope of $\Psi_{\text {ad all }}$ was almost zero, weak $P s u$ fluctuations began to come out. These fluctuations occurred at all the Psu measurement positions and the peak frequencies were $19 \%$ of the impeller rotation frequency. Then, as the flowrate was slightly reduced, the positive slope part of $\Psi_{\text {ad_all }}$ appeared. At the operating point [e], where the slope of $\Psi_{\text {ad_all }}$ was already positive, the amplitudes of the fluctuations repeated to become larger and smaller (Points [e]-1 and [e]-2). In addition, intermittent spike-like fluctuations, as indicated by dashed arrows in Fig. 8, were also observed at the LSD1, LSD2, and LSD3. It was confirmed that these spike-like fluctuations propagated in the direction from the LSD1 to the LSD3, which was the same direction as the impeller rotation. At the safety operation limit [f], the amplitudes of the fluctuations became much larger and the peak frequencies fell from approximately $19 \%$ to $9.4 \%$ of the impeller rotation frequency. When the valve was closed slightly more, periodic Psu fluctuations with the largest amplitude at this IOR occurred. The peak frequencies of these fluctuations were $9.3 \%$ of the impeller rotation frequency. In the lower flowrate than the operating point [d], a lot of jagged waveforms were superimposed on the periodic pressure fluctuations with the peak frequency of about $9 \%$ of the impeller rotation frequency that were observed at the vaneless space. Each of these jagged waveforms was considered to correspond to the spike-like fluctuation.

\subsection{Measurement results at IOR 4.5\%}

Figures 10 and 11 show measured Psu fluctuations at IOR 4.5\%.

At the largest operating point [g] in Fig. 5, no clear Psu fluctuations were observed. However, when the operating point reaches the point [h], weak $P s u$ fluctuations appeared. The peak frequencies of these fluctuations were $22.9 \%$ of the impeller rotation frequency. At the operating point [i], the amplitudes of these $P s u$ fluctuations repeated to become larger and smaller (Points [i]-1 and [i]-2). The peak frequencies of these fluctuations were $21.8 \%$ of the impeller rotation frequency, slightly lower than those at the operating point [h]. These fluctuations were observed at all the $P s u$ measurement positions. At this operating point, the inception of the intermittent spike-like fluctuations, as indicated by dashed arrows in Fig.10, were also observed at the LSD1, LSD2, and LSD3 (Point [i]-2). Like the case of the operating point [e], these spike-like fluctuations propagated in the direction of the impeller rotation. At the operating point [j], the amplitudes of the Psu fluctuations became much larger. The peak frequencies of these fluctuations became lower compared to those at the operating point [i], i.e., 19.1\% of the impeller rotation frequency. At the smallest flowrate point $[\mathrm{k}]$, the amplitudes of the fluctuations did not change so much compared to those at the operating point [j], however the peak frequencies fell to $14.4 \%$ of the impeller rotation frequency. In the lower flowrate than the operating point [i], jagged waveforms, as with the case at IOR 10\%, were superimposed on the periodic pressure fluctuations that were observed at the vaneless space. Each of these jagged waveforms was also considered to correspond to the spike-like fluctuation. At this IOR, it should be noticed that the slope of $\Psi_{\text {ad_all }}$ remained steeply negative throughout the measured operating point.

\subsection{Discussion on causes of $\boldsymbol{P} \boldsymbol{s} \boldsymbol{u}$ fluctuations}

As mentioned above, we confirmed several types of pressure fluctuation in the low flowrate region.

The first fluctuation had relatively large amplitude and the peak frequency of approximate seven to nine percent of the impeller rotation frequency. This was observed at all the $P s u$ measurement positions and appeared when the operating point exceeded the safety operation limit at IOR $100 \%$ and $10 \%$, where the slope of $\Psi_{\text {ad_all was flat or }}$ positive. In general, it is said that surge occurs where the slope of the system head-flow characteristic becomes positive. Therefore, this fluctuation is considered to be surge.

According to past literatures, at least four different categories of surge can be distinguished with respect to flow and pressure fluctuations: Mild surge, classic surge, deep surge, and modified surge (de Jager, 1995). Mild surge is a phenomenon with small pressure fluctuation and no reverse flow. Mild surge is essentially a Helmholtz oscillator and the peak frequency of mild surge is governed by the Helmholtz resonance frequency. Classic surge is a phenomenon that has larger pressure fluctuation and lower frequency than those of mild surge. Modified surge is a situation where rotating stall and surge exist together. Deep surge is a more severe version of classic surge, where even reverse flow is possible. Greitzer showed the shapes of limit cycle and the time histories of the Psu fluctuation during classic surge and 

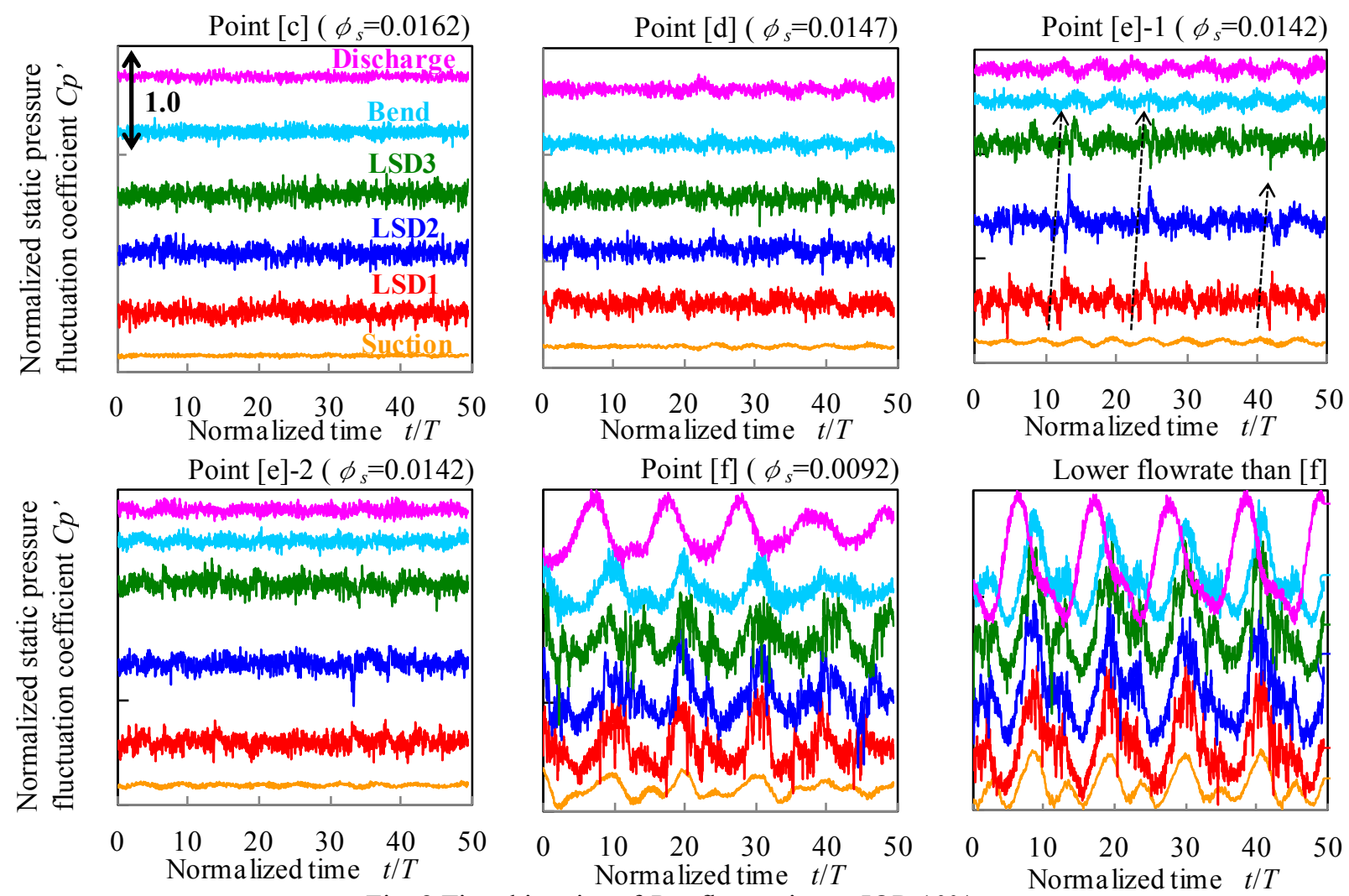

Fig. 8 Time histories of $P s u$ fluctuation at IOR $10 \%$.
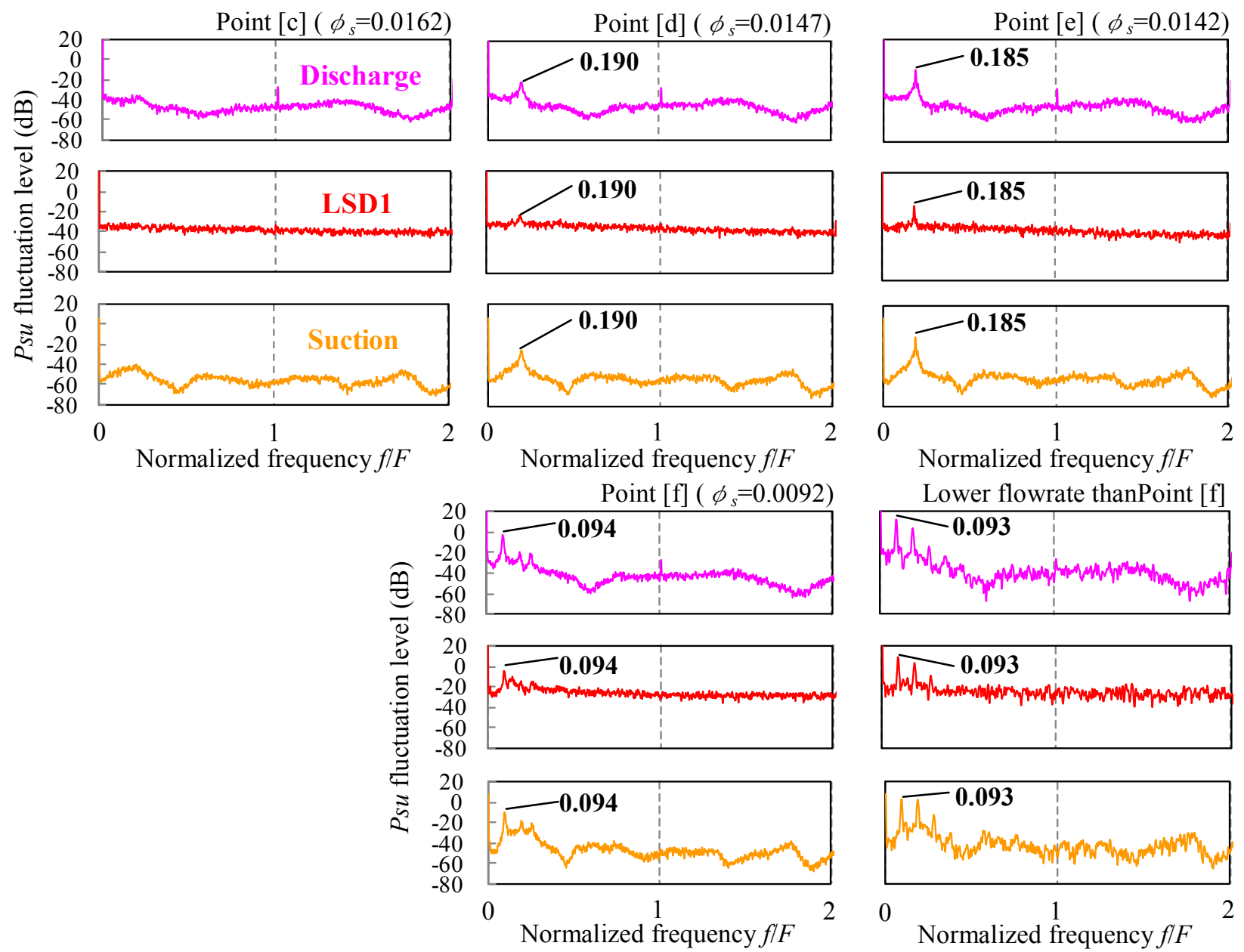

Normalized frequency $f / F$

Lower flowrate thanPoint [f]
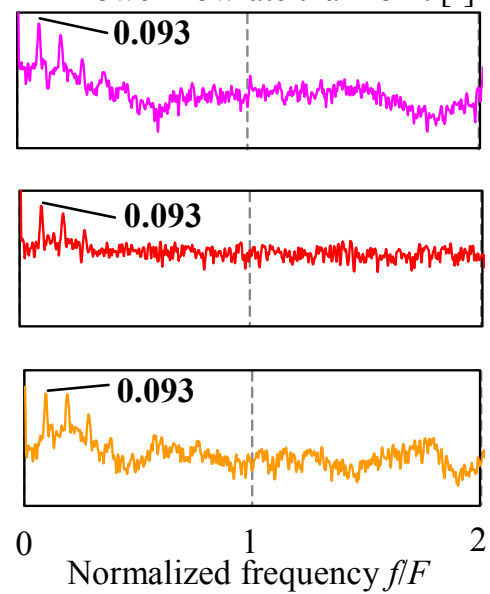

Fig. 9 Time-averaged $P s u$ fluctuation spectra at IOR 10\%. 

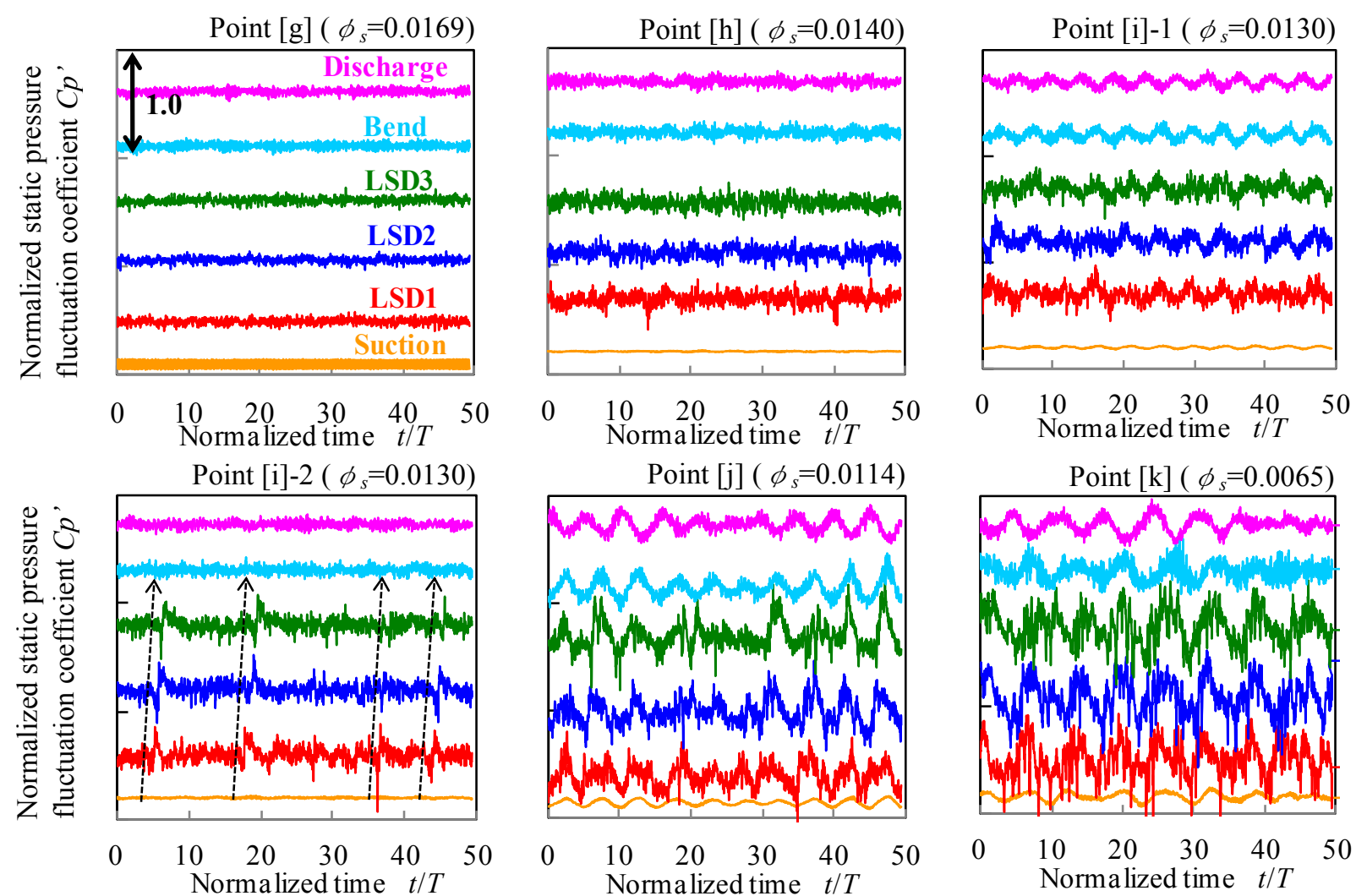

Fig. 10 Time histories of $P s u$ fluctuation at IOR 4.5\%.
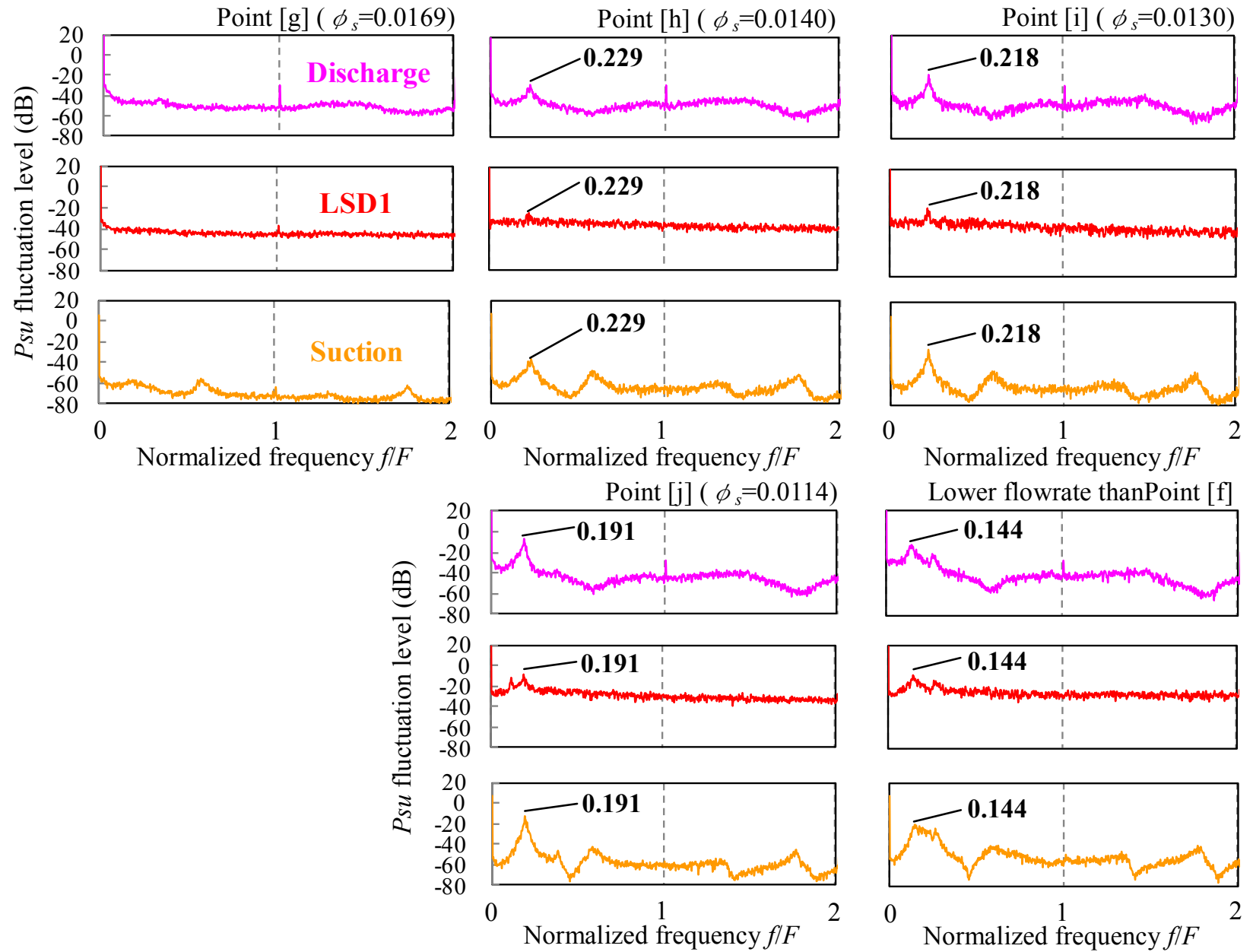

Normalized frequency $f / F$

Lower flowrate thanPoint [f]
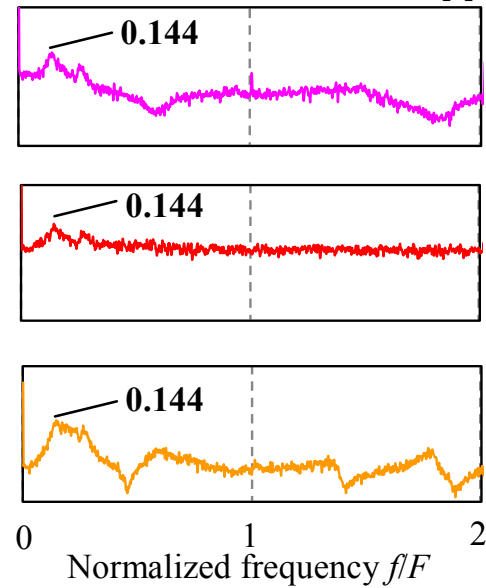

Fig. 11 Time-averaged $P s u$ fluctuation spectra at IOR 4.5\%. 
deep surge in an axial compressor (Greitzer, 1976b). According to this study, the shape of the limit cycle is nearly circular and the waveform of the $P s u$ fluctuation is relatively sinusoidal during classic surge. Meanwhile, the shape of the limit cycle becomes rather squarish and crests and troughs of the $P s u$ waveform become sharper during deep surge. Since we did not conduct an unsteady flowrate measurement, we could not clarify whether the reverse flow occurred during surge. However, as shown in Figs. 6 and 8, the waveform of the first fluctuation was sharp and similar to that during deep surge shown by Greitzer. Therefore, we considered this fluctuation to be deep surge.

The second fluctuation was the spike-like fluctuation intermittently observed only at the pressure transducers LSD1, LSD2, and LSD3. This occurred in the lower flowrate region than the operating point [d] at IOR $10 \%$ and the operating point $[\mathrm{h}]$ at IOR $4.5 \%$. Since this fluctuation definitely propagated in the circumferential direction, this was considered to be short wavelength rotating stall similar to that observed by Spakovszky et al. and Joukou et al. (Spakovszky and Roduner, 2007, Joukou, et al., 2012). As will be discussed later in this section, this rotating stall was thought to occur in all stages at not only IOR 10\%, where the slope of $\Psi_{\text {ad_all }}$ was positive, but also IOR $4.5 \%$ condition, where the slope of $\Psi_{\text {ad_all }}$ was steeply negative.

The last fluctuation was the sinusoidal fluctuation observed at all the sensors with smaller amplitude and higher frequency than those of the first fluctuation. This occurred from the operating point [d] to [f] at IOR 10\% and in lower flowrate region than the operating point $[\mathrm{h}]$ at IOR 4.5\%. In case at IOR 10\%, since this fluctuation occurs in the region where the slope of $\Psi_{\text {ad_all }}$ was flat or positive, a cause of the fluctuation was considered to be surge. Since the amplitude of this fluctuation was much smaller than that of the first fluctuation, we considered this fluctuation to be mild surge. In the lower flowrate than the operating point [d], the sinusoidal fluctuation with spike-like rotating stall in the vaneless space was observed. This was thought to be modified surge.

On the other hand, this sinusoidal fluctuation occurred in the region where the slope of $\Psi_{\text {ad_all }}$ was steeply negative at IOR 4.5\%. We conducted the cross spectrum analysis of Psu time history between LSD1 and LSD3 to elucidate the cause of this fluctuation. Figure 12 shows a result of time-averaged cross spectrum analysis at the operating point [i]-1. The horizontal axis means normalized frequency $f / F$ and the vertical axis means cross spectrum and phase difference spectrum. The black line represents the cross spectrum and the red line represents the phase difference spectrum. As shown in Fig. 12, the cross spectrum between LSD1 and LSD3 had the peak at 21.8\% of the impeller rotation frequency. This peak frequency was the same as that observed at the suction nozzle and the discharge scroll at the same operating point. Moreover, the phase difference between LSD1 and LSD3 was zero at this peak frequency. Therefore, the cause of this fluctuation was considered not to be rotating stall, but to be also mild surge. Moreover, this fluctuation must be said to be modified surge since mild surge observed in lower flowrate than that at the operating point [h] was accompanied by spike-like rotating stall in the vaneless space.

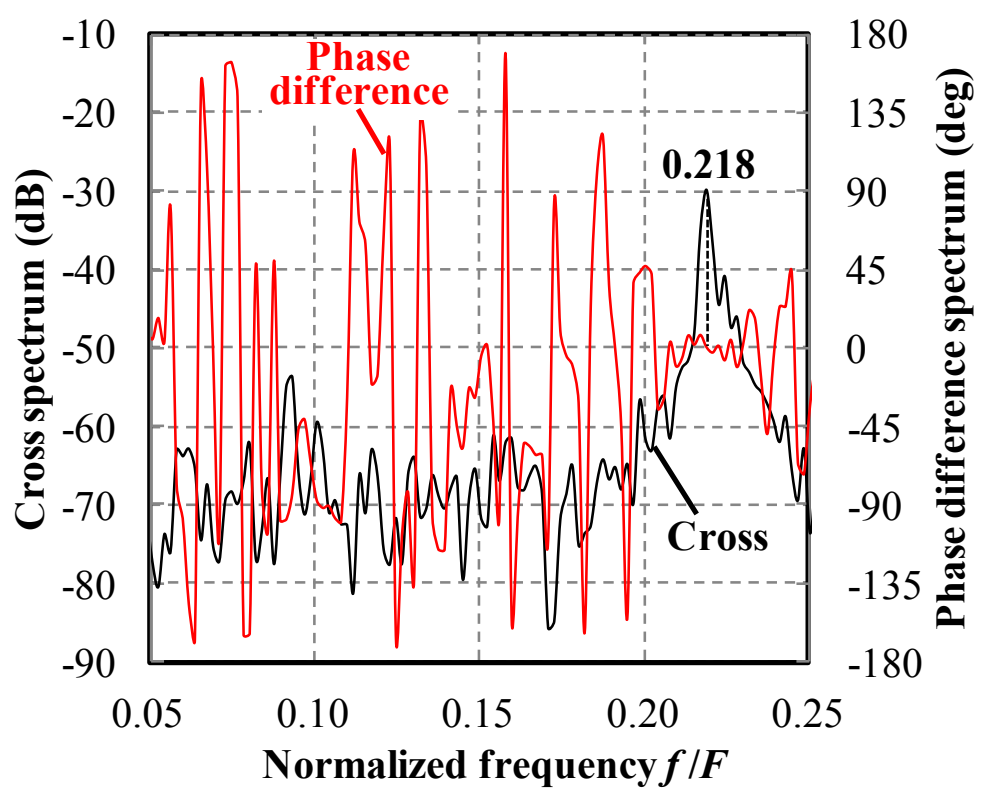

Fig. 12 Result of cross spectrum analysis between LSD1 and LSD3. 
We had thought that the whole compression system was basically stable and surge did not occur in the negative slope region of $\Psi_{\text {ad_all }}$. However, it was clarified that mild surge could occur even in the steeply negative slope region of $\Psi_{\text {ad_all }}$. We considered the reason why mild surge could occur in the negative slope region of $\Psi_{\text {ad_all }}$ to be as follows.

Figure 13 shows the static pressure-rise characteristics of the first and second stage at IOR 10\%. The black squares correspond to $C p$ in the first stage and the white squares correspond to $C p$ in the second stage. As shown in Fig. 13, while $C p$ in the first stage kept the steeply negative slope to the same flowrate as the operating point [i], where the mild surge became remarkable at IOR 4.5\%. This was because the strong prewhirl was given to the impeller in the first stage by the IGVs. In contrast, the slope of $C p$ in the second stage became flat around the operating point [d], where the slope of $\Psi_{\text {ad_all }}$ became nearly zero and mild surge began to appear at IOR 10\%. As shown in Fig. 5, the suction flow coefficient at the safety operation limit [b] at IOR 100\% was almost the same as that at the inception point [d] of mild surge at IOR $10 \%$. Since no intentional prewhirl was given to the second and the subsequent stages and each stage was designed to satisfy the similarity condition of the inner flow, the stall inception flowrates of these stages were thought to be almost the same regardless of IOR condition (Strictly speaking, the stall inception flowrates of these stages at IOR $10 \%$ is supposed to become slightly smaller than those at IOR 100\% since the pressure-rise in the first stage decreases due to the increase in the prewhirl and the pressure loss given by the IGVs).

We were not able to obtain $C p$ characteristic curves at IOR $4.5 \%$ since yaw-angle and/or pitch-angle measured by the pitot-tubes exceeded the calibration range. However, it is thought that similar $C p$ characteristic curves to those at IOR $10 \%$ are also obtained at IOR $4.5 \%$. That is, while $C p$ of the first stage keeps steeply negative slope at least to the operating point [i], the second and the subsequent stages begin to stall at slightly smaller flowrate than that corresponds to the operating point [d]. At IOR 4.5\%, the negative slope of $C p$ of the first stage is considered to be much steeper than that at IOR $10 \%$. Therefore, even if the second and the subsequent stages stall, the steeply negative slope of $\Psi_{a d}$ all maintained, and the total system seems to be kept stable. However, the slopes of the pressure-rise characteristics of the second and the subsequent stages are already positive. Therefore, these stages should destabilize the system and lead the system to much deeper surge condition with larger oscillation of the operating point. On the other hand, the strong stabilization effect of the first stage ought to prevent the system from falling into deeper surge condition. We considered that mild surge at IOR $4.5 \%$ was caused by these stabilization and destabilization effects. That is, this was the situation when the system operating point oscillated slightly due to a balance of these stabilization and destabilization effects.

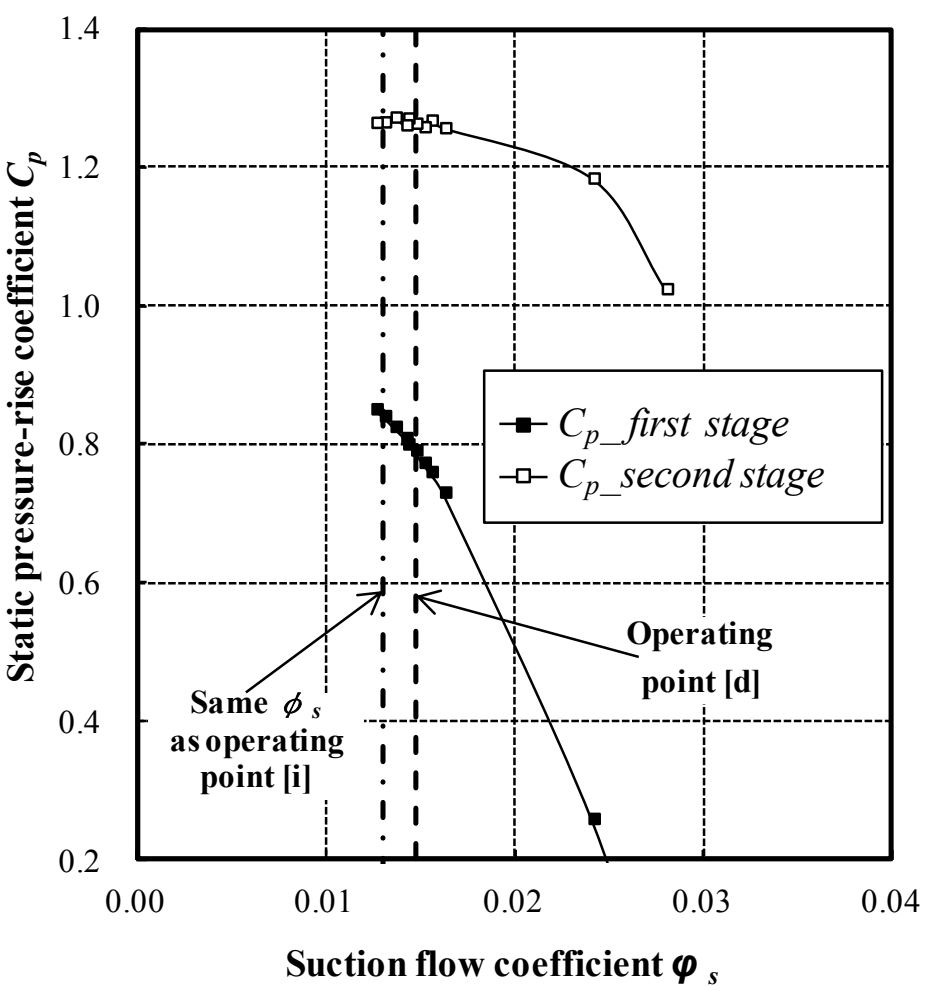

Fig. 13 Static pressure-rise characteristics of first and second stage at IOR 10\% 
At IOR $100 \%$, only the deep surge occurred in this blower. In blowers with relatively low pressure ratio and no prewhirl, when the blowers are designed so that all stages satisfy the similarity condition of the inner flow, each stage is thought to stall and enter the unstable operation region at almost the same time. In this situation, since there is no element to stabilize the system, the perturbation of the operating point is amplified rapidly. Therefore, it was considered that only the deep surge was observed under IOR 100\% condition in this blower. Since the blower operated under IOR $100 \%$ condition is almost equivalent to that with no IGVs, it is supposed that only the deep surge should be also observed in case with no IGVs. On the contrary, both types of surge, i.e., deep surge and mild surge, occurred under partial IGV opening conditions, where the influence of IGVs on the blower performance became more apparent. It can be said that the installation of IGVs could change the surge characteristic from deep surge to mild surge.

As mentioned above, the spike-like rotating stall was observed in the second stage diffuser at the operating point [e] at IOR $10 \%$ and the operating point [i] at IOR 4.5\%. In this blower, all blower stages were designed to satisfy the similarity condition of the inner flow. Therefore, the rotating stall must have occurred in the diffusers at the second and subsequent stages at these operating conditions. On the other hand, it was considered that this rotating stall should have also occurred in the first stage diffuser. This is because even if the large prewhirl was given to the first stage impeller under these IOR conditions, the average absolute flow angle at the impeller outlet of the first stage was thought to be almost the same compared to those of the other stages. The spike-like rotating stall is thought to be caused by the propagation of the leading edge separation vortex in the circumferential direction (Pullan, et al, 2012). Therefore, it is considered to be valid that the leading edge separation vortex occurred in the first stage diffuser, as with the other stages. As a result, the spike-like rotating stall should have also occurred in the first stage diffuser despite the steeply negative slope of the first stage characteristic due to the prewhirl at these operating points.

\section{Conclusion}

We carried out the detailed measurements of the unsteady pressure fluctuation in the five-stage centrifugal blower with IGVs upstream of the first stage to clarify unstable flow phenomena occurred in the low flowrate region. The findings obtained in this study were summarized as follows:

(1) We confirmed three kinds of pressure fluctuations in low flowrate region in this blower.

(2) The first fluctuation had relatively large amplitude. The peak frequency of this fluctuation was about $7-9 \%$ of the impeller rotation frequency. This was observed throughout the blower and occurred when the operating point exceeded the safety operation limit at IOR $100 \%$ and $10 \%$, where the slope of $\Psi_{\text {ad_all }}$ was flat or positive. This fluctuation was considered to be deep surge.

(3) The second fluctuation was the spike-like fluctuation intermittently observed only in the vaneless space between the impeller outlet and the LSD leading edge. This occurred at IOR 10\% and IOR 4.5\% and propagated in the circumferential direction. This was thought to be short wavelength rotating stall.

(4) The last fluctuation was the sinusoidal fluctuation observed throughout the blower with smaller amplitude and higher frequency than that of the first fluctuation. This occurred at IOR $10 \%$ and IOR $4.5 \%$. At IOR $10 \%$ condition, this began to appear from the flat slope region of $\Psi_{\text {ad_all }}$ and was considered to be mild surge. On the other hand, this also occurred even in the steeply negative slope region of $\Psi_{\text {ad }}$ all in case at IOR $4.5 \%$. We considered this fluctuation at IOR $4.5 \%$ to be also mild surge caused by the balance of the stabilization effect due to the steeply negative slope of the first stage characteristic and the destabilization effect due to the positive slope of the second-last stage characteristics.

\section{References}

Arnulfi, G., L., Micheli, D., and Pinamonti, P., 1996, Experimental investigation on rotating stall in a centrifugal blower with two and four stages and vaneless diffusers, ASME 1996 International Gas Turbine and Aeroengine Congress and Exhibition (1996), Paper No. 96-GT-171.

Arnulfi, G., L., Giannattasio, P., Giusto, C., Massardo, A., F., Micheli, D., and Pinamonti, P., Multistage centrifugal compressor surge analysis: Part I - Experimental investigation, ASME Journal of Turbomachinery, Vol.121, No.2 (1999), pp.305-311. 
Arnulfi, G., L., Giannattasio, P., Giusto, C., Massardo, A., F., Micheli, D., and Pinamonti, P., Multistage centrifugal compressor surge analysis: Part II - Numerical simulation and dynamic control parameters evaluation, ASME Journal of Turbomachinery, Vol.121, No.2, pp.312-320.

de Jager, B., Rotating stall and surge control : A survey, Proceedings of the $35^{\text {th }}$ IEEE Conference on Decision and Control (1995), pp.1857-1862.

Fink, D., A., Cumpsty, N., A., and Greitzer, E., M., Surge dynamics in a free-spool centrifugal compressor system, ASME Journal of Turbomachinery, Vol.114, No.2 (1992), pp.321-332.

Greitzer, E., M., Surge and rotating stall in axial flow compressors, Part I: Theoretical compression system model, ASME Journal of Engineering for Power, Vol.98, No.2 (1976), pp.190-198.

Greitzer, E., M., Surge and rotating stall in axial flow compressors, Part II: Experimental results and comparison with theory," ASME Journal of Engineering for Power, Vol.98, No.2 (1976), pp.199-211.

Hansen, K., E., Jørgensen, P., and Larsen, P., M., Experimental and theoretical study of surge in a small centrifugal compressor, ASME Journal of Fluids Engineering, Vol.103, No.3 (1981), pp.391-395.

Joukou, S., Shinkawa, Y., Kanno, T., Nishida, H., Nishioka, T., and Hiradate, K., Influence of low-solidity cascade diffuser on spike stall inception in a centrifugal compressor, ASME Turbo Expo 2012 (2012), Paper No. GT-2012-69203.

Lüdtke, K., 1992, The influence on adjustable inlet guide vanes on the perfomance of multi-stage industrial centrifugal compressors, ASME 1992 International Gas Turbine and Aeroengine Congress and Exposition (1992) ASME Paper No. 92-GT-17.

Pullan, G., Young, A. M., Greitzer, E. M., and Spakovszky, Z. S., Origins and Structure of Spike-Type Rotating Stall, ASME Turbo Expo 2012 (2012), Paper No. GT-2012-68707.

Senoo, Y., Kinoshita, Y., and Ishida, M., Asymmetric flow in vaneless diffusers of centrifugal blowers, ASME Journal of Fluids Engineerign, Vol.99, No.1 (1977), pp.104-111.

Senoo, Y., Kinoshita, Y., Limits of rotating stall and stall in vaneless diffuser of centrifugal compressors, ASME International Gas Turbines Conference and Products Show (1978), Paper No. 78-GT-19.

Spakovszky, Z. S., and Roduner, C. H., Spike \& modal stall inception in an advanced turbocharger centrifugal compressor, ASME Turbo Expo 2007 (2007), Paper No. GT-2007-27634. 